\title{
Brain observations
}

\author{
New tools are improving the prospects for transcranial light-based neuroscience, but \\ better methods for using them are needed before they can reach their full potential.
}

One year ago on this page, we highlighted advances in the development of genetically encoded fluorescent probes used to monitor neuronal activity at the level of single cells. The ability to express these probes in vivo in targeted neuronal populations is opening up new possibilities for studying the fine-scale functioning of the brain. But full exploitation of existing tools is hampered by protective systems, such as the blood-brain barrier and skull, that shield the brain from external assaults, including those coming from curious neuroscientists.

Introduction of fluorescent probes or optogenetic tools by viral transduction-a popular method for obtaining high-level expression in neurons-necessitates physically breaching the skull to inject the virus into a region of interest. Last month, Nature Methods published a promising alternative devised by Louboutin et al. that yielded efficient viral delivery of genes into mature neurons in the brain using less invasive intravenous injection of virus and mannitol. Unfortunately, packaging of the recombinant viral vectors used in this study inactivated fluorescent proteins through a still unknown mechanism. Before the method can fulfill its potential, it will be crucial to overcome this limitation.

But even if fluorescent probes are delivered without opening the skull, high-resolution fluorescence microscopy cannot penetrate the native skull to image single neurons. Researchers can mechanically thin the bone in a small region of the skull until it is thin enough to image through, but poor light penetration and bone regrowth-requiring re-thinning for chronic imaging - limit image quality and number of imaging sessions. Consequently, researchers often prefer the cranial window technique using a glass window over a hole in the skull, despite the need to wait $\sim 2$ weeks for the inflammatory response to subside before imaging.

Groups using the cranial window and thinned skull methods have reported contradictory data that some have attributed to the techniques. These contradictory results have already spurred investigators to take more care in their technique and perform important controls, and considerable progress has been achieved in standardizing and improving the cranial window method. But these necessary precautions are costly: it often takes six months of training before users become adept at performing the technique.

On page 981, Kleinfeld and colleagues describe an alternative: the polished and reinforced thinned skull (PoRTS) procedure provides a larger field of view and better optical clarity than the original thinned skull method and allows unlimited imaging sessions over three months-with no sign of inflammatory response. The availability of a third method that combines advantages of the existing ones provides a valuable opportunity for the neuroscience community.

A third choice for optical access to the brain may help resolve conflicting results from the other techniques and provides researchers with flexibility in their experimental design. With PoRTS, once the-admittedly considerable-time and effort is expended on preparing the window, the possibility of immediate imaging access and unlimited imaging sessions makes a variety of investigations possible.

As neuroscientists conduct ever more complex behavioral experiments that require substantial time and effort to properly train mice and rats, there is increasing need for techniques that allow chronic transcranial microscopy. No method yet performs well enough that it will be clearly preferred over the others for every application; researchers will need to weigh the advantages and disadvantages of each. Because they limit the number of potentially confounding experimental variables, the difficulty and cost of implementing less invasive methods may be fully justified even if they require extra effort.

Despite recent advances, the holy grail of noninvasive cellular-level functional perturbation and imaging of the entire brain is still a long way off. New optics techniques and development of red-shifted probes and optogenetic tools will be needed to increase the depths that can be transcranially sampled, and new procedures must be developed to create clearer noninvasive cranial windows.

Moreover, even with such developments, light-based transcranial imaging will almost certainly be limited to 1-1.5 mm from the surface of the brain. These depths are sufficient for answering many questions, but for sampling the full brain of mice and other larger animals, a new generation of probes and tools-perhaps based on magnetism and paramagnetic particles-will be needed. Until then, better procedures and experimental methods will be necessary for light-based probes to reach their full potential in providing insight into the function of the intact brain. 